\title{
PET systems: the value of added length
}

\author{
Giovanni Borasi - Federica Fioroni • \\ Alberto Del Guerra • Giovanni Lucignani
}

Published online: 26 March 2010

(C) Springer-Verlag 2010

The past decade has brought unbelievable advances in CT technology. These have been reflected in a progressive widening of the radiation beam in the axial direction (from "fan beam" to "cone beam" geometry) and in a corresponding increase in the number of detector rings.

From the four detectors and $2 \mathrm{~cm}$ axial field of view (AFOV), acquired in half a second, that characterised the systems of a decade ago, we now have, with the present technology, 256/312 multi-row detectors that allow the acquisition of an $8 / 16 \mathrm{~cm}$ axial field in less than a third of a second. In addition, dual-source CT is offering a wealth of new possibilities.

These new systems, whose realisation demanded the solution of complex mechanics, electronics and information technology problems, have opened up a completely new

G. Borasi $(\bowtie) \cdot$ F. Fioroni

Medical Physics Department, Santa Maria Nuova Hospital,

V. le Risorgimento 80,

42100 Reggio Emilia, Italy

e-mail: giovanni_borasi@libero.it

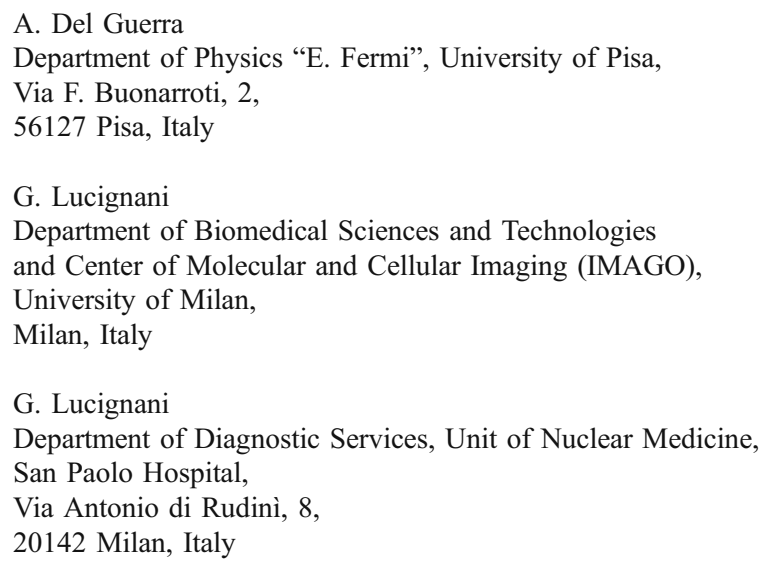

A. Del Guerra

Department of Physics "E. Fermi", University of Pisa,

Via F. Buonarroti, 2,

56127 Pisa, Italy

G. Lucignani

Department of Biomedical Sciences and Technologies and Center of Molecular and Cellular Imaging (IMAGO), University of Milan,

Milan, Italy

\section{G. Lucignani}

Department of Diagnostic Services, Unit of Nuclear Medicine,

San Paolo Hospital,

Via Antonio di Rudinì, 8,

20142 Milan, Italy

field of medical investigation: the four-dimensional study of the heart and the vascular system.

The past few years have also seen evolutions in the field of PET/CT. Indeed, this combined modality has benefitted not only from improved CT technology, but also from gradual advances in the PET sphere-advances prompted by the introduction of new crystal detectors (such as LSO, GSO, LYSO), new iterative reconstruction algorithms, faster electronics, the "gating" technique (synchronisation of data acquisition with respiratory motion) and, especially, "time of flight" PET technology (TOF) [1-4].

Yet despite these significant improvements, the length of the detector cylinder, or length of the system's sensitive volume ("field of view"), has remained essentially the same $(15-18 \mathrm{~cm})$. With this acquisition geometry, only a very small fraction of the radiation emitted at any given instant is acquired (geometric efficiency $\leq 0.2$ ). Therefore, to obtain, for example, a "whole-body" study of about $80 \mathrm{~cm}$ in length (from the temples to the bladder of the patient), a series of six to eight static scans ("views") need to be acquired. The adjacent single views, obtained with the movement of the bed, must, of course, also provide an appropriate region of overlap. Obviously, the acquisition time increases in proportion to the number of views. A "standard study" with the injection of $10 \mathrm{mCi}$ of ${ }^{18} \mathrm{~F}-\mathrm{FDG}$ takes about $3 \mathrm{~min}$ per view and, therefore, approximately 25 min per entire study. To this about a minute should be added for the CT scan. However, when used for wholebody studies in diagnostic oncology - this use accounts for approximately $90 \%$ of all PET scans-this procedure presents major limitations. The limited length of the sensitive volume not only imposes the need for multiple views, and thus prolongs the overall duration of the examination, it also (and this is particularly important) limits the maximum "line of response" (LOR) angle (with 
respect to the radial direction of the system), that is the line of flight of the pair of photons arising from a single annihilation event.

PET sensitivity increases as the square of the LOR angle (a fact whose importance became very clear when we moved from 2-D to 3-D acquisition). Therefore, leaving aside the intrinsic efficiency of the detectors and the electronics, the main cause of the system's low global efficiency lies in the reduced value of the acceptance angle (between 2 and $7 \%$, for a point source placed at the centre of the field of view). The drawbacks of this low global efficiency-low image quality, long scan times and the need for high radiopharmaceutical doses-translate into low productivity, high costs per single study and a substantial reduction in the range of possible applications of the technique. Long scan times also favour the generation of artefacts due to possible movements of the patient, especially in CT fusion studies. Similarly, the overlapping of subsequent views can easily give rise to artefacts in organs included in more than one field of view. An even more serious limitation is the lack of quantitative evaluations: the fact that different body regions are acquired at different times practically rules out the possibility of performing dynamic (tracer time course) studies.

\section{New extended field of view geometry: the challenges}

In short, PET systems with an extended AFOV $(\geq 90 \mathrm{~cm})$ would be the ideal solution to a wide range of problems. However, to be of practical value, these systems must solve the new problems, both from the system physics and cost point of view, raised by this geometry. As regards the first of these aspects, it must be appreciated that AFOV increases are accompanied by increases in the number not only of true coincidence events (being revealed), but also of spurious events (random or due to scattered radiation). In addition, when the angle at which the photon reaches the detector increases, so too does the probability that the photon will be intercepted by two or more crystals, making its location imprecise and reducing the quality of the image (parallax effect or "crosstalk"). This is, indeed, a major problem and one that is exacerbated by the highly asymmetric shape of crystal detectors, which is due to the need to achieve, simultaneously, high spatial resolution and high detection efficiency. This problem, which already afflicts standard length PET scanners, can be resolved by the use of detectors, possibly as a columnar structure, able to identify the depth within the crystal at which the interaction took place (depth of interaction, DOI). Electronic detection could, of course, prove to be a critical element given that it has to manage a photon flux that grows more than proportionately to the length of the AFOV. The major problem, however, is the cost of the system that, in PET, is largely ascribable (about $40-60 \%)$ to the volume of the crystal scintillators used.

\section{First proposals and prototypes}

Is there concrete evidence of an evolution of PET towards systems with longer fields of view? Siemens recently proposed a PET/CT system that increases the number of detector rings from three to four, extends the AFOV from 16 to $22 \mathrm{~cm}$ and implements TOF technology.

Developments of this kind can probably be considered part of the dawn of a new era, which began a decade ago with the study by Badawi et al. [5] on the effect of PET geometry on PET system performance. In this study, which took the characteristics of the GE Advance PET system as reference, the effects of increasing both AFOV (from 15 to $60 \mathrm{~cm}$ ) and detector ring diameter (DRD) (from about 60 to $108 \mathrm{~cm}$ ) were simulated on an anthropomorphic phantom with Monte Carlo code (1 m scan length, $10 \mathrm{mCi}$ of FDG administered, waiting time of $1 \mathrm{~h}$ ). At a constant DRD, an increase in AFOV from 15 to $60 \mathrm{~cm}$ allows a noise equivalent count (NEC) growing by a factor of about 7 . In the same conditions, when AFOV increases between 15 and $30 \mathrm{~cm}$, the scatter increases from 30 to $34 \%$ and then it remains practically at the same value up to $60 \mathrm{~cm}$ length. Tomograph performances decreased significantly with reduction of the DRD: the correlated scatter increase is responsible for this not so obvious result. The maximum count rate was close to $100 \mathrm{MHz}(250 \mathrm{MHz}$ assuming the case of isotopes with shorter half-life than ${ }^{18} \mathrm{~F}$ ), a result considered difficult but not impossible to achieve with the technology of the time.

The first proposal for a truly "total-body" PET system (120-160 cm AFOV) came from an Italian physicist, Crosetto [6]. The first two prototypes that subsequently emerged can be seen to be moving in opposite directions in terms of philosophy and approach: the Knoxville-based Siemens Medical Solutions group [7, 8] developed a tomograph (P39 5H) based on large LSO "flat panels": $53 \mathrm{~cm}$ in the axial direction and $37 \mathrm{~cm}$ in the transaxial direction, assembled to form five sides of a hexagon in constant rotation (30 rpm). Each panel consists of 70 block detectors and each block is a $12 \times 12$ array of $4 \times 4 \times 20 \mathrm{~mm}^{3}$ crystals. The sixth side contains the system for transmission measurements. This is a cost-effective geometry that makes it possible to reduce the number of photomultipliers by a factor of 4 . The system has an axial sensitivity of $2 \%$, measured with a $70 \mathrm{~cm}$ source (NU2-2001); the scatter fraction is $40 \%$ and the spatial resolution is approximately $5 \mathrm{~mm}$. In clinical conditions, the system operates at a count rate lower than $5 \mathrm{MHz}$. Only two views (10 min each) are needed for a complete scan $(80 \mathrm{~cm})$. 
In the study mentioned above [8], the prototype was compared directly with the HI-REZ in two patients. The prototype performs two 10-min views (total $20 \mathrm{~min}$ ), $90 \mathrm{~min}$ after injection, reconstruction algorithm OP-OSEM3D, while the HI-REZ performs seven 2-min views (total $14 \mathrm{~min}$ ) $140 \mathrm{~min}$ after injection, reconstruction algorithm not specified. A 3-D Gaussian filter is applied to both images. The first patient $(60 \mathrm{~kg})$ was administered $10.1 \mathrm{mCi}$ of activity and the second $(107 \mathrm{~kg})$, who underwent a similar protocol, received $12.2 \mathrm{mCi}$. The prototype images showed lower resolution and higher noise than those produced by the commercial model, but the prototype (by definition) should have room for improvement.

The second prototype (SHR-92000), which has a $68.5 \mathrm{~cm}$ AFOV, was made by the Japanese group Hamamatsu [9]. The detection system consists of 12 layers of block detector rings stacked axially, each ring consisting of a circular array of 60 block detectors. Each block detector consists of a newly designed scintillation position-sensitive plate photomultiplier, which subtends a $16 \times 8$ BGO crystal array, each one measuring $2.9 \times 6.3 \times 20 \mathrm{~mm}^{3}$. To reduce the scatter fraction, metal septa are positioned between different adjacent rings (5-mm thick, 180-mm long). These septa do not appear in the image, since the patient bed, in performing an acquisition, continuously scans a distance equal to one pitch of the septa $(57.6 \mathrm{~mm})$. This is therefore an intermediate geometry, somewhere between 2-D and 3-D (2.5-D). The system has an axial sensitivity of $0.97 \%$ (NU2-2001) and a scatter fraction of $31.4 \%$, and the NEC peak is $113.6 \mathrm{kcps}$ (at $10.5 \mathrm{kBq} / \mathrm{ml}$ ). No data regarding spatial resolution are available. In this case too, the full scan of the patient requires only two $10-\mathrm{min}$ views: the clinical protocol provides for the injection of $3.4 \mathrm{mCi}$ for a standard patient and a waiting time of $65 \mathrm{~min}$. According to the authors, the resulting image showed very high sharpness, but "vignetting" due to the movement of the septa appears clearly detectable in the presented image.

\section{New detectors: the challenges}

The successful development of a PET system with an extended AFOV clearly depends very much on progress made in the field of detector technology. In this regard, the "resistive plate chamber" (RPC) technology, derived from high energy physics, is a particularly promising development. RPC detectors, which can be produced in large plates, have high time, spatial and energy resolution and are relatively inexpensive. They also allow evaluation of the DOI, thereby solving the parallax problem. The high time resolution also allows the implementation of TOF techniques. One limitation, however, is their rather low intrinsic sensitivity, which is resolved by creating, in the radial direction, a "sandwich" of several plates (60-120). Using this technology, researchers at the Coimbra University in Portugal [10-13], after creating a high-resolution prototype for animal studies, are now working on a $2 \mathrm{~m}$-long totalbody prototype, at the end of which is mounted a small CT system. Assuming an acceptance angle of $30^{\circ}$, a length of $2 \mathrm{~m}$ and the presence of 120 plates, it is estimated that sensitivity can be increased, compared with the GE Advance system, by a factor of 20 (including a factor of 4.4 due to TOF). The estimated scatter fraction is $46 \%$ and the cost of the final system should not exceed that of a conventional PET system.

\section{Cost or quality?}

The possibilities offered by increasing the length of the field of view, well beyond that suggested by Badawi [5], were recently analysed by Eriksson et al. [14]. They evaluated the "sensitivity profile", i.e. the response of detectors in the axial direction when a very long thin rectilinear source is placed on the axis. They evaluated the current Siemens models (HI-REZ: three rings of detectors, $16.4 \mathrm{~cm}$ field of view; and TruePoint: four rings of detectors, $22 \mathrm{~cm}$ field of view). Both systems use LSO crystals. With the first scanner, seven views are needed to cover a $70 \mathrm{~cm}$ axial field, whereas with the second, five views are sufficient. From the three-ring $(16.4 \mathrm{~cm}$ field of view) to the four-ring system (22 cm field of view), sensitivity increases by a factor of about 2 . This "gain" is reflected in a reduction of the time needed for each view (from 180 to $137 \mathrm{~s}$ ) and in improved "image quality". The time needed for the whole scanning procedure (product of time for each view by number of views) is reduced by a factor of about 2, corresponding to the increase in absolute sensitivity.

The authors also considered two hypothetical models with more detector rings (10 and 20 rings corresponding to a length of 55.6 and $111.6 \mathrm{~cm}$, respectively), assuming their use with the current technology (and angle of acceptance). With the longer model, the whole scan (39 s) is accomplished with a single view, and the sensitivity is increased by a factor of 30 compared with the three-ring system and by a factor of 17 compared with the four-ring system. It is important to note that the performances of these systems are calculated without the use of TOF, which further increases the sensitivity by a factor of 2-4. With this latest technology and the $112 \mathrm{~cm}$-long system, it can be assumed that a single scan would take just 8-20 s. This means that the entire examination could be carried out within the space of a breath-hold, thereby giving the added advantage of eliminating the significant blurring effect related to respiratory motion. If we consider that the intrinsic spatial 
resolution in these conditions is less than a millimetre, this can certainly be seen as a revolutionary development.

However, the financial aspect cannot be ignored. Considering, as mentioned earlier, that the cost of the crystals accounts for $40-60 \%$ of the total cost, a large field of view system would carry a price tag of around 5-6 million euros, compared with the (approximately) 2 million euro price tag of current scanners, and this is without considering the additional costs due to the considerable increase in technological complexity.

So should we be looking for cheaper solutions that do not have recourse to high energy physics technologies?

In a recent paper Eriksson et al. [15] evaluated the performance of a hypothetical system based on the same LSO crystals technology used in their previous study [14]; in this case, however, the crystal thickness was reduced (4.3 versus $20 \mathrm{~mm}$ ). The $196 \mathrm{~cm}$-long tomograph would allow the body to be scanned, with a single view, in $5.8 \mathrm{~min}$, as opposed to the $7.5 \mathrm{~min}$ taken by the TruePoint TrueV system with its $22 \mathrm{~cm}$-long axial field of view, five views and $20 \mathrm{~mm}$-thick crystals. The "long" system has been shaped to provide approximately the same sensitivity as both the "short" system and an RPC system of the same length. The cost of this thin crystals system should not exceed the cost of current systems. Another "economical" solution, based on existing technology (BGO), is proposed by Wai-Hoi et al. [16]. This system, with its $100 \mathrm{~cm}$ AFOV, consists of 275 rings, each with 748 crystals. Each BGO crystal is $3.5 \times 3.5 \times 20 \mathrm{~mm}^{3}$ in size and the DRD is $82.5 \mathrm{~cm}$. Compared with the GE Advance, this system reduces the examination time by a factor of 6 and the dose of by a factor of 4 . The cost of producing the system, analysed in detail in the study, would be US $\$ 1,250,000$, of which less than half (US $\$ 500,000$ ) is due to the cost of the crystals (compared with US \$2,000,000 using LYSO).

\section{But can we now do without the benefits of TOF?}

In a recent study, Poon et al. [17] developed a detailed simulation, based on anthropomorphic phantoms, of LSO tomographs up to $2 \mathrm{~m}$ long. With very fast electronics (several hundred MHz) a $2 \mathrm{~m}$-long system should be able to increase sensitivity by a factor of 25-50, compared with the "reference" GE Discovery STE system.

At this point the question to be answered is: is it better to exploit the TOF technology to further increase the signal to noise ratio by a factor between 2 and 3 (creating, as seen above, a high-cost system) or, instead, to "settle for" a factor of 25-50 and spend the TOF benefits to concentrate on reducing the thickness of the crystals, thereby developing a relatively low-cost system.

Whichever option prevails, it seems likely that we will see, in the not too distant future, extended field of view
PET scanners, with sensitivity increased by more than one order of magnitude.

Acknowledgement The authors are grateful to Ms. Catherine Wrenn for her advice and skilful editorial support.

\section{References}

1. Cherry SR. The 2006 Henry N. Wagner Lecture: Of mice and men (and positrons) - advances in PET imaging technology. J Nucl Med 2006;47(11):1735-45.

2. Townsend DW. Multimodality imaging of structure and function. Phys Med Biol 2008;53(4):R1-39.

3. Townsend DW. Dual-modality imaging: combining anatomy and function. J Nucl Med 2008;49(6):938-55.

4. Conti M. State of the art and challenges of time-of-flight PET. Phys Med 2009;25(1):1-11.

5. Badawi RD, Kohlmyer SG, Harrison RL, Vannoy SD, Lewellen TK. The effect of camera geometry on singles flux, scatter fraction and trues and randoms sensitivity for cylindrical 3D PET-a simulation study. IEEE Trans Nucl Sci 2000;47(3):1228-32.

6. Crosetto DB. A modular VME or IBM PC based data acquisition system for multi-modality PET/CT scanners of different sizes and detector types. Presented at the IEEE Nuclear Science Symposium and Medical Imaging Conference, Lyon, France, 2000, Published in the Conference Record, http://www.3d-computing.com/pb/ ieee2000563.pdf.

7. Jones WF, Reed J, Everman J, Luk P, Gremillion T, Castleberry B, et al. First time measurement of transaxial resolution for a new high-sensitivity PET prototype using 5 LSO panel detectors. IEEE Nucl Sci Symp Conf Rec 2002;2:694-698.

8. Conti M, Bendriem B, Casey M, Eriksson L, Jakoby B, Jones WF, et al. Performance of a high sensitivity PET scanner based on LSO panel detectors. IEEE Trans Nucl Sci 2006;53(3):1136-42.

9. Watanabe M, Shimizu K, Omura T, Sato N, Takahashi M, Kosugi $\mathrm{T}$, et al. A high-throughput whole-body PET scanner using flat panel PS-PMTs. IEEE Trans Nucl Sci 2004;51:796-800.

10. Blanco A, Carolino N, Correia CM, Fazendeiro L, Ferreira NC, Marques MF, et al. RPC-PET: a new very high resolution PET technology. IEEE Trans Nucl Sci 2006;53(5):2489-94.

11. Couceiro M, Blanco A, Ferreira NC, Ferreira Marques R, Fonte P, Lopes L. RPC-PET: status and perspectives. Nucl Instrum Methods Phys Res A 2007;580(2):915-8.

12. Couceiro M, Ferreira NC, Fonte P. Sensitivity assessment of wide axial field of view PET systems via Monte Carlo simulations of NEMA-like measurements. Nucl Instrum Methods Phys Res A 2007;580(1):485-8.

13. Blanco A, Couceiro M, Crespo P, Ferreira NC, Ferreira Marques R, Fonte P, et al. Efficiency of RPC detectors for whole-body human TOF-PET. Nucl Instrum Methods Phys Res A 2009;602(3):780-3.

14. Eriksson L, Townsend D, Conti M, Eriksson M, Rothfuss H, Schmand $M$, et al. An investigation of sensitivity limits in PET scanners. Nucl Instrum Methods Phys Res A 2007;580(2):836-42.

15. Eriksson L, Townsend DW, Conti M, Melcher CL, Eriksson M, Jakoby BW, et al. Potentials for large axial field of view positron camera systems. IEEE Nucl Sci Symp Conf Rec 2008;2008:1632-6.

16. Wai-Hoi W, Yuxuan Z, Hongdi L, Ramirez H, Jiguo R, Liu S, et al. The initial design and feasibility study of an affordable high-resolution 100-cm long PET. IEEE Nucl Sci Symp Conf Rec 2007;6:4117-4122

17. Poon JK, MacDonald LR, Cherry SR, Badawi RD. A simulation study of a long axial field of view whole body PET scanner using cylindrical and anthropomorphic phantoms. IEEE Nucl Sci Symp Conf Rec 2008:M10-266. 